\title{
Wavelet Packet Methods for the Analysis of Variance of Time Series With Application to Crack Widths on the Brunelleschi Dome
}

\author{
Francesco GabBANINI, Marina VANNUCCI, \\ Gianni BARTOLI, and Antonio MORO
}

\begin{abstract}
In this article we extend the definition of wavelet variance to wavelet packets. We also adapt to wavelet packets an iterated cumulative sum of squares algorithm for the location of variance change points. Wavelet packets have greater decorrelation properties than standard wavelets in that they induce a finer partitioning of the frequency domain of the process generating the data. This allows our procedure to be applied to a wide class of processes. We show this on simulated data and on a benchmark time series. Our initial interest in wavelet variance change points location was motivated by an application to time series of crack widths on the Brunelleschi dome of the Santa Maria del Fiore cathedral in Florence. The structure of the dome includes an internal thick dome and an external thin one. In an effort to understand the dynamics of the crack widths we apply wavelet packet variance analysis to measurements from instruments located in the different parts of the outer and inner domes, highlighting different features and seasonal behavior. Our findings agree well with the structural functions of the different elements of the dome and also reveal some interesting aspects regarding the dynamics of crack evolution.
\end{abstract}

Key Words: Change points detection; Nondecimated transforms; Wavelet variance.

Note: Supplementary material for this article is available on the World Wide Web at http://www.amstat.org/publications/jcgs/ftp.html.

\footnotetext{
Francesco Gabbanini, Department of Statistics, University of Florence, viale Morgani 59, 50134 Florence, Italy, (E-mail: gabbanin@ds.unifi.it). Marina Vannucci, Department of Statistics, Texas A\&M University, 3143 TAMU, College Station, TX 77843-3143 (E-mail: mvannucci@ stat.tamu.edu). Gianni Bartoli, Department of Civil Engineering, University of Florence, via di S. Marta 3, 50139 Florence, Italy (E-mail: gbartoli @ dicea.unifi.it). Antonio Moro, Department of Civil Engineering, University of Florence, via di S. Marta 3, 50139 Florence, Italy (E-mail: moro@dicea.unifi.it).
}

\section{(c)2004 American Statistical Association, Institute of Mathematical Statistics, and Interface Foundation of North America}

Journal of Computational and Graphical Statistics, Volume 13, Number 3, Pages 639-658

DOI: $10.1198 / 106186004 X 2372$ 


\section{INTRODUCTION}

The wavelet variance was first introduced by Percival (1995) as a tool for the decomposition of the variance of a time series into different components, each associated with a particular scale (or resolution). Percival proved that the wavelet variance estimator is unbiased and asymptotically Gaussian when the underlying process is Gaussian. Serroukh, Walden, and Percival (2000) extended these results to the case of various nonlinear, nonGaussian and stationary or locally stationary processes.

Given a time series $\left\{Y_{t}, 0 \leq t \leq N-1\right\}$, one of the main conditions for the wavelet variance estimator to be unbiased is that the process $\left\{Y_{t}\right\}$ must be $I(d)$, that is, its $d$ th order backward difference must be a stationary process. With real time series, however, it is not uncommon to encounter departures from this assumption. For instance, in physical sciences atmospheric variables often show increasing variation in certain months of the year. In such cases, one possible approach is to use testing procedures to identify variance change points, therefore locating intervals where the variance can be considered constant. Wavelet variances can then be computed in each of these intervals. Inclàn and Tiao (1994) proposed an iterated cumulative sum of squares (ICSS) algorithm to identify multiple change points in the variance of a series of independent observations. Whitcher et al. $(2000,2002)$ applied this algorithm to coefficients from standard discrete wavelet transforms. However, since the procedure requires independent observations, they restricted themselves to data from long-memory processes for which one can assume approximately uncorrelated wavelet coefficients.

In this article we explore some new techniques based on wavelet packet transforms. We first define a wavelet variance estimator based on wavelet packets. In the supplementary material we show how statistical properties of the wavelet variance easily extend to packets. We then investigate the use of the wavelet packet variance in the analysis of time series with nonconstant variance via the ICSS algorithm. We show how the employment of wavelet packets makes this algorithm more flexible than when standard wavelets are used. Wavelet packets, in fact, induce a finer partitioning of the frequency space, implying better decorrelation properties than the DWT, and therefore allowing the algorithm to perform well for a wide class of processes that might have generated the data. We exploit performances of the proposed methods on simulated data, with both single and multiple change points, by computing empirical size and power of the testing procedure. We apply the method to a time series of subtidal coastal sea levels previously analyzed with wavelet variance. In the examples we use the classical Ljung-Box test for autocorrelation (Box, Jenkins, and Reinsel 1994) to exploit correlation at the different packet levels. Examples show the great potential of wavelet packet variance techniques as exploratory tools for time series analysis.

We conclude the article with an application to time series of crack widths on the Brunelleschi dome of Santa Maria del Fiore cathedral in Florence. The structure of the dome includes an internal thick dome and an external thin one. We analyze measurements from instruments located in different parts of the outer and inner domes. Wavelet analysis highlights a significant seasonal behavior in the variance of the time series measured by instruments located in the internal dome of the cathedral, whereas the dynamic of crack 
widths measured by instruments located in the inner dome appears to be different, mainly because of the absence of any seasonality in the variance. A possible explanation is in the different structural functions that the two domes have. Wavelet analysis also captures the action of the side chapels that greatly limit the evolution of cracks, reflecting in a smaller wavelet variance for measurements coming from instruments located at the bottom of the dome.

The article is organized as follows: Section 2 reviews the most important concepts regarding standard wavelet transforms and wavelet variance. Section 3 briefly introduces discrete wavelet packet transforms and defines the wavelet packet variance as a generalization of the wavelet variance. Section 4 outlines the ICSS algorithm applied to wavelet packet variance. Section 5 reports simulation studies and real examples and Section 6 concludes the article. Theoretical results are shown in the supplementary material available on the World Wide Web.

\section{PRELIMINARIES}

\subsection{The Maximal Overlap Discrete Wavelet Transform}

The maximal overlap discrete wavelet transform (MODWT) of Percival and Walden (2000) is basically a nondecimated version of the discrete wavelet transform (DWT) of Mallat (1989). The level- $j$ MODWT of a time series $\left\{Y_{t}, 0 \leq t \leq N-1\right\}$ is defined by circular linear filtering as

$$
\tilde{W}_{j, t}=\sum_{l=0}^{L_{j}-1} \tilde{h}_{j, l} Y_{(t-l) \bmod N}, \quad 0 \leq t \leq N-1
$$

with $\tilde{h}_{j, l}=h_{j, l} / 2^{j / 2}$ and $\left\{h_{j, l}\right\}$ representing a level- $j$ wavelet filter of length $L_{j}=\left(2^{j}-\right.$ $1)(L-1)+1$ with $L$ the width of the wavelet filter (on unit scale). Filter coefficients vary according to the wavelet family. Here we are concerned with Daubechies (1992) wavelets, which have compact support, implying filters with a finite number of nonzero coefficients. Coefficients $\left\{\tilde{W}_{j, t}\right\}$ represent differences between generalized averages of the time series $\left\{Y_{t}\right\}$ on a scale $\tau_{j}=2^{j-1}$ (or level $j$ ). Unlike the case of the DWT, MODWT coefficients at each level are not subsampled. Nondecimated transforms with strong analogies with the MODWT were defined by Shensa (1992), Coifman and Donoho (1995), and Nason and Silverman (1995).

\subsection{The Wavelet Variance}

Let $\left\{Y_{t}, t \in \mathbb{Z}\right\}$, with $\mathbb{Z}$ the set of integer numbers, be a discrete parameter real-valued stochastic process. By filtering $\left\{Y_{t}\right\}$ with a level- $j$ MODWT wavelet filter of length $L_{j}$ we 
obtain the stochastic process

$$
\bar{W}_{j, t}=\sum_{l=0}^{L_{j}-1} \tilde{h}_{j, l} Y_{t-l}, \quad t \in \mathbb{Z} .
$$

The time-dependent wavelet variance of $\left\{Y_{t}\right\}$ at level $j$ is then defined as

$$
\nu_{Y, t}^{2}(j)=\operatorname{var}\left\{\bar{W}_{j, t}\right\}
$$

In practice, an estimate is obtained by assuming that the variance of $\left\{\bar{W}_{j, t}\right\}$ is constant over time, therefore defining

$$
\nu_{Y}^{2}(j)=\operatorname{var}\left\{\bar{W}_{j, t}\right\}
$$

and an unbiased estimate is constructed using the MODWT coefficients of Equation (2.1) that are not affected by the modulus operation as

$$
\hat{\nu}_{Y}^{2}(j)=\frac{1}{M_{j}(N)} \sum_{t=L_{j}-1}^{N-1} \tilde{W}_{j, t}^{2}
$$

with $M_{j}(N)=N-L_{j}+1$. Here, following Percival and Walden (2000), we restrict ourselves to processes such that the wavelet variance exists, is finite and independent of $t$. It is then possible to prove that the asymptotic distribution of $\hat{\nu}_{Y}^{2}(j)$ is Gaussian, a result that allows the formulation of confidence intervals for the estimate. See Percival (1995) and Serroukh, Walden, and Percival (2000) for methods and proofs.

\section{PACKET VARIANCE}

\subsection{The Discrete Wavelet Packet Transform}

The discrete wavelet packet transform (DWPT) of a time series $\left\{Y_{t}\right\}$ is a generalization of the DWT. At the first level of the transform a low and a high filter are applied to $\left\{Y_{t}\right\}$ to obtain, after subsampling, $\left\{W_{1,0, t}\right\}$, corresponding to the frequency band $[0,1 / 4]$, and $\left\{W_{1,1, t}\right\}$, corresponding to the frequency band $[1 / 4,1 / 2]$. At level $j$ the same steps are repeated by filtering and subsampling the low passed and high passed coefficients obtained at the previous level. Here we refer to what Wickerhauser (1994) calls the "sequency order." The rule for such ordering is that at any level $j$, given $\left\{W_{j, n, t}\right\}$, the filters get swapped when $n$ is odd, that is, the high-pass filter is used to obtain $\left\{W_{j+1,2 n, t}\right\}$ and the low-pass filter is used to obtain $\left\{W_{j+1,2 n+1, t}\right\}$. This order differs from the "natural order," where for every $n$ low-pass filter and high-pass filter are used to obtain $\left\{W_{j+1,2 n, t}\right\}$ and $\left\{W_{j+1,2 n+1, t}\right\}$ from $\left\{W_{j, n, t}\right\}$, respectively. See Wickerhauser (1994) for more details. The sequency order is physically useful, in that at any level $j$ a larger frequency index $n$ corresponds to increasing the center of the corresponding frequency band. The natural order, however, requiring fewer 
computations, is often the choice adopted by software packages, such as the Matlab Wavelet Toolbox we used in the analyses. From a computational point of view the DWPT can be readily computed by a very simple modification of the pyramid algorithm introduced by Mallat (1989) for the DWT.

The maximal overlap discrete wavelet packet transform (MODWPT) is basically a nondecimated version of the DWPT, introduced by Walden and Contreras Cristan (1998). As a filtering of the original time series it can be written as

$$
\tilde{W}_{j, n, t}=\sum_{l=0}^{L_{j}-1} \tilde{f}_{j, n, l} Y_{(t-l) \bmod N}, \quad 0 \leq t \leq N-1
$$

for $n=0, \ldots, 2^{j}-1$, where

$$
\tilde{f}_{j, n, l}=\sum_{k=0}^{L-1} \tilde{f}_{n, k} \tilde{f}_{j-1,\lfloor n / 2\rfloor, l-2^{j-1} k}, \quad 0 \leq l \leq L_{j}-1
$$

with

$$
\tilde{f}_{n, l}=\left\{\begin{array}{cccc}
\tilde{g}_{l} & \text { if } & n \bmod 4=0 & \text { or } 3 \\
\tilde{h}_{l} & \text { if } & n \bmod 4=1 & \text { or } 2
\end{array}\right.
$$

and $\tilde{g}_{l}=(-1)^{l+1} \tilde{h}_{L-l-1}$, and such that $\left\{\tilde{f}_{1,0, l}=\tilde{g}_{l}, 0 \leq l \leq L-1\right\}$ and $\left\{\tilde{f}_{1,1, l}=\tilde{h}_{l}, 0 \leq\right.$ $l \leq L-1\}$. For additional details see Walden and Contreras Cristan (1998).

\subsection{The Wavelet Packet Variance}

Following the same approach described for the wavelet variance we now introduce the wavelet packet variance based on the nondecimated discrete wavelet packet transform (MODWPT). Given $\left\{Y_{t}, t \in \mathbb{Z}\right\}$ a discrete parameter real-valued stochastic process, we therefore define the $[j, n]$ packet variance $\nu_{Y, t}^{2}(j, n)$ as the variance of $\bar{W}_{j, n, t}$, if it exists and is finite,

$$
\nu_{Y, t}^{2}(j, n)=\operatorname{var}\left\{\bar{W}_{j, n, t}\right\}
$$

with

$$
\bar{W}_{j, n, t}=\sum_{l=0}^{L_{j}-1} \tilde{f}_{j, n, l} Y_{t-l}, n>0, \quad t \in \mathbb{Z} .
$$

We again restrict ourselves to processes such that the wavelet variance exists, is finite and independent of $t$. An unbiased estimate of $\nu_{Y}^{2}(j, n)$ that uses the MODWPT coefficients in Equation (3.1) can then be obtained from filters that do not overlap the ends of the data as

$$
\hat{\nu}_{Y}^{2}(j, n)=\frac{1}{M_{j}(N)} \sum_{t=L_{j}-1}^{N-1} \tilde{W}_{j, n, t}^{2} .
$$


In the supplementary material we show that the wavelet packet variance estimator is unbiased and asymptotically Gaussian for Gaussian data. Proofs of these results can be readily obtained along the lines of those proved for the wavelet variance by Percival (1995). Also, Serroukh et al. (2000) have generalized some results about the distribution of the wavelet variance estimator to processes that are not necessarily Gaussian. Their results are fairly general with respect to the wavelet filters, and we expect similar extensions to be possible for the wavelet packet variance.

\section{TESTING FOR VARIANCE CHANGES}

Both the estimator (2.5) of the wavelet variance and the one (3.6) of the packet variance are based on the hypothesis that the variance of the underlying stochastic process is constant. When this is not the case, testing procedures can be used to identify variance change points by locating intervals where the variance can be considered constant. Wavelet variance values can then be computed in each of these intervals.

The problem of identifying multiple change points in the variance of a sequence of independent observations has been addressed by several authors. Inclàn and Tiao (1994) proposed a procedure based on an iterated cumulative sum of squares algorithm. Whitcher et al. (2000, 2002) applied the ICSS procedure to wavelet coefficients obtained from the DWT and did extensive simulation studies for both single and multiple change points. Because the procedure requires independent coefficients, they assumed data from long memory processes, specifically stationary Gaussian fractionally differenced. Decorrelation properties of the DWT for such processes are, in fact, well studied—see, for example, Tewfik and Kim (1992) — and allow one to assume wavelet coefficients at a given level as approximately uncorrelated.

\subsection{Detection and Location of Multiple Variance Changes via Wavelet PACKeTS}

We have investigated the use of the ICSS algorithm with wavelet packet coefficients. The adaptation of the procedure to packets is fairly straightforward. Wavelet packets, however, induce a finer partitioning of the frequency space, implying greater flexibility and better decorrelation properties than the DWT, therefore allowing the algorithm to perform well for a wider class of processes than the long memory. Intuitively, at level $j$ a wavelet packet transform partitions the frequency interval $[0,1 / 2]$ into $2^{j}$ equal intervals of the form

$$
\left[\frac{n}{2^{j+1}}, \frac{n+1}{2^{j+1}}\right], \quad n=0,1, \ldots, 2^{j}-1,
$$

and we can therefore expect that a process with a spectral density function relatively flat in any of the intervals of the partition would result in approximately uncorrelated coefficients at the corresponding packet. The standard wavelet transform, on the other hand, produces, at level $j$, a single set of coefficients associated with the interval $\left[1 / 2^{j+1}, 1 / 2^{j}\right]$, and in this 
sense it is less flexible. Decorrelation properties of the wavelet packets have been exploited by Percival, Sardy, and Davison (2000) who proposed an adaptive wavelet-based scheme for bootstrapping statistics from time series as realizations of some long and short memory processes.

Before reporting on examples let us briefly summarize the test procedure of Inclàn and Tiao (1994) and discuss its adaptation to wavelet packet coefficients. Let $\left\{Y_{t}, 0 \leq t \leq\right.$ $N-1\}$ be a finite realization of a sequence of uncorrelated random variables with zero means and variances $\sigma_{0}^{2}, \sigma_{1}^{2}, \ldots, \sigma_{N-1}^{2}$. We want to test the hypothesis $H_{0}: \sigma_{0}^{2}=\cdots=\sigma_{N-1}^{2}$ against the alternative $H_{1}: \sigma_{0}^{2}=\cdots=\sigma_{k}^{2} \neq \sigma_{k+1}^{2}=\cdots=\sigma_{N-1}^{2}$. The test uses a normalized cumulative sum of squared test statistic $D=\max \left(D^{+}, D^{-}\right)$where

$$
D^{+}=\max _{0 \leq k \leq N-2}\left(\frac{k+1}{N-1}-P_{k}\right), \quad D^{-}=\max _{0 \leq k \leq N-2}\left(P_{k}-\frac{k}{N-1}\right),
$$

and $P_{k}=\left(\sum_{j=0}^{k} Y_{j}^{2}\right) /\left(\sum_{j=0}^{N-1} Y_{j}^{2}\right)$. When testing for multiple change points the ICSS algorithm iteratively computes $D$ on subseries obtained from $\left\{Y_{t}\right\}$. Critical levels for $D$ under the null hypothesis can be obtained via Monte Carlo simulation. Inclàn and Tiao (1994) also proved that the asymptotic distribution of the statistic is one of a Brownian bridge. This allows one to perform the test using an asymptotic approximation for the critical values of $D$ when the sample size is at least 128 .

In our adaptation to wavelet packets, we first select a wavelet packet and then perform the ICSS algorithm with the selected coefficients. The selection of the wavelet packet is done by increasing the level of the DWPT transform until the Ljung-Box test fails to reject the hypothesis of white noise for at least one set of coefficients. If more than one set of coefficients falls into this category we select the one with the smallest value of the LjungBox test statistics. We also typically repeat the procedure for different wavelet filters, again preferring the filter that gets smaller values of the test statistic. For the selected packet we compute the test statistic $D$ using the DWPT wavelet packet coefficients, after correcting for phase effects and discarding the coefficients that involve the use of circularity. Phase effects were fully investigated by Wickerhauser (1994) and Walden and Contreras Cristan (1998). The location of the variance change points is estimated with the nondecimated packet transform as

$$
\hat{k}=\operatorname{argmax}(D),
$$

with $D$ computed on the MODWPT coefficients.

\section{APPLICATIONS}

This section shows possible situations where the estimation of variance change points requires the use of the DWPT-based procedure. We then discuss an application to a time series of subtidal coastal sea levels previously investigated by Percival and Mofjeld (1997) 


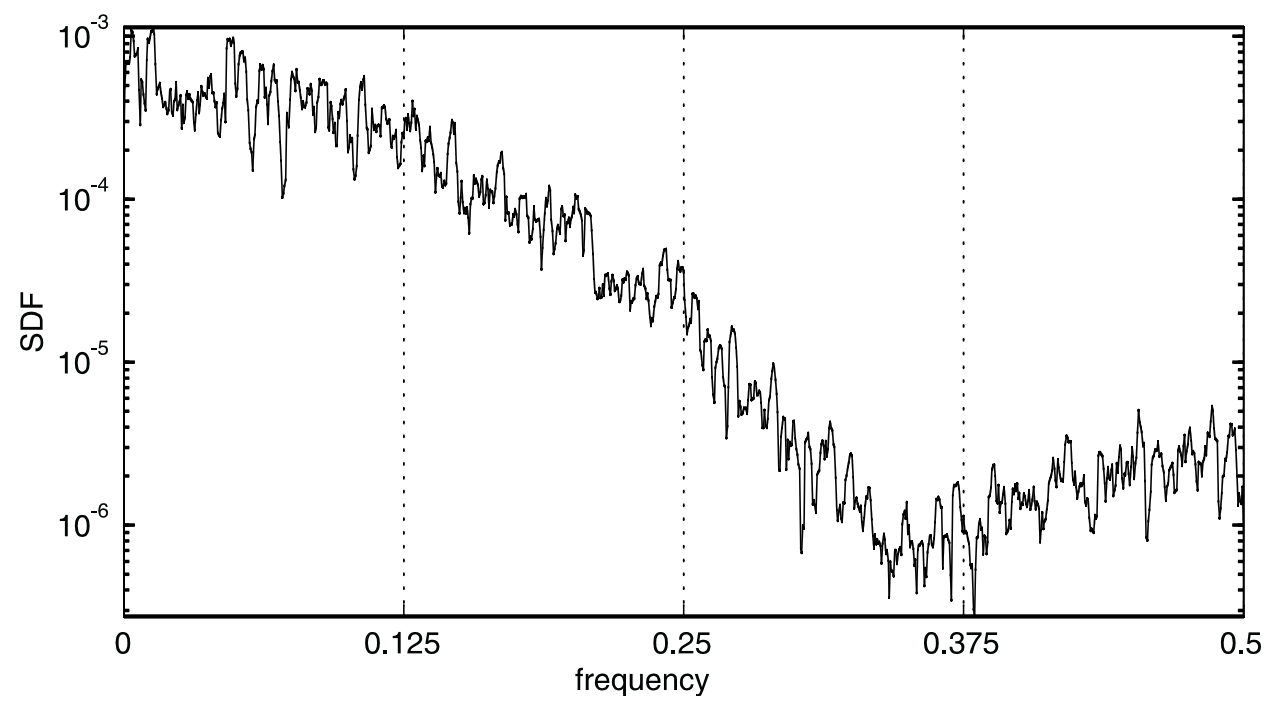

Figure 1. ARMA(2,2): Spectral density function estimate of process (5.1). Vertical dotted lines identify frequency bands that correspond to packets [2,0], [2,1], [2,2], and [2,3], respectively. Packet [2,1] corresponds to the level 2 DWT wavelet coefficients. Packets are computed in "sequency order."

with the DWT. We conclude with the analysis of a time series of crack widths on the Brunelleschi dome in Florence.

In our analyses we use Daubechies (1992) wavelets, and specifically the so-called extremal phase $\mathrm{D}(L)$ and the least asymmetric $\mathrm{LA}(L)$ with filter length $L$. We refer interested readers to Daubechies (1992) for a description of these filters. In their simulation studies, on fractionally differenced processes only, Whitcher et al. $(2000,2002)$ used three different wavelet families-Haar, D(4), and LA(8). They found that power and size of the ICSS algorithm decrease with the wavelet level and recommended not to use levels greater than three. They also found very little differences in power and size among the different wavelet filters they used. In our study we investigate performances of the ICSS procedure on wavelet packet coefficients from processes different from the long memory and make use of the Ljung-Box test for the presence of autocorrelation to test whether the packet transform produces uncorrelated coefficients at a given packet. In the following, we report on results with the filters that best decorrelate the data.

\subsection{Simulation Study}

Let $\left\{X_{t}\right\}$ be an $\operatorname{ARMA}(2,2)$ stochastic process defined as

$$
\left(1-0.7 B+0.2 B^{2}\right) X_{t}=\left(1+0.9 B+0.6 B^{2}\right) \epsilon_{t},
$$

where $B$ is the backward shift operator, $B X_{t}=X_{t-1}$, and with $\epsilon_{t} \sim N\left(0, \sigma^{2}\right)$. An estimate of the spectral density function (SDF) of this process is shown in Figure 1. The SDF is not approximately constant over either of the frequency intervals associated with the first two 

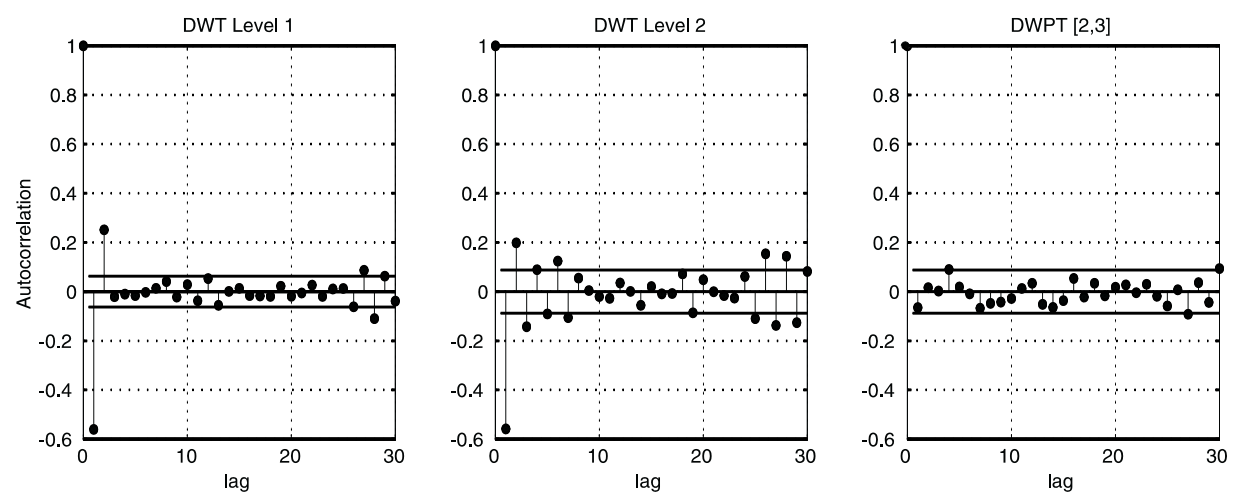

Figure 2. ARMA(2,2): Autocorrelations of level 1 DWT coefficients, level 2 DWT coefficients, and packet [2,3] DWPT coefficients, from a realization of process (5.1) with $N=2,048$.

DWT levels, namely $[0.25,0.5]$ and $[0.125,0.25]$. We thus cannot expect to obtain time series of uncorrelated DWT wavelet coefficients. This is confirmed by the autocorrelation functions of the coefficients at the first two DWT levels shown in Figure 2 and by the LjungBox test, in Table 1, performed with a 5\% significance level at lags 10, 20, and 30, using least asymmetric wavelets with filters of length 8 (Daubechies 1992). Better results are instead obtained with wavelet packets—-see again Figure 2 and Table 1 - and indeed coefficients at packet $[2,3]$ can be considered uncorrelated. In the "sequency order" the wavelet packet $[2,3]$ corresponds to the frequency band $[0.375,0.5]$, where indeed the spectral density in Figure 1 appears to be reasonably flat.

For a better appreciation of the consequences of having uncorrelated wavelet coefficients, let us now examine the empirical size and power of the ICSS algorithm for both the DWT and DWPT. Figure 3 shows rejection rates obtained by simulating, for each $N=2^{j}, 5 \leq j \leq 11,10,000$ realizations of process (5.1), with constant variance, and by performing the test with Monte Carlo critical values with significance levels $\alpha=0.01$ and $\alpha=0.05$. When one uses DWT coefficients the presence of correlation results in an over-rejection of the (true) null hypothesis of constant variance. At DWT levels 1 and 2 rejection rates are well in excess of $10 \%$ with $\alpha=0.05$ (see the lines marked with squares and diamonds in the plot on the left) and above $5 \%$ with $\alpha=0.01$ when $N \geq 128$ (plot on the right). When, instead, the $D$ statistic is computed with packet [2,3] DWPT coefficients

Table 1. ARMA(2,2): Ljung-Box $Q$ Test Statistic Values for Level 1 DWT Coefficients, Level 2 DWT Coefficients and Packet [2,3] DWPT coefficients, from a realization of process (5.1) with $N=$ $2,048.5 \%$ critical values are in parentheses.

\begin{tabular}{ccll}
\hline \hline Level & \multicolumn{1}{c}{$Q_{10}$} & \multicolumn{1}{c}{$Q_{20}$} & \multicolumn{1}{c}{$Q_{30}$} \\
\hline 1 & $337.06(18.31)$ & $349.41(31.41)$ & $358.81(43.77)$ \\
2 & 169.39 & 174.48 & 185.57 \\
2,3 & $\mathbf{9 . 2 9}$ & $\mathbf{1 2 . 9 2}$ & $\mathbf{1 6 . 7 5}$ \\
\hline
\end{tabular}



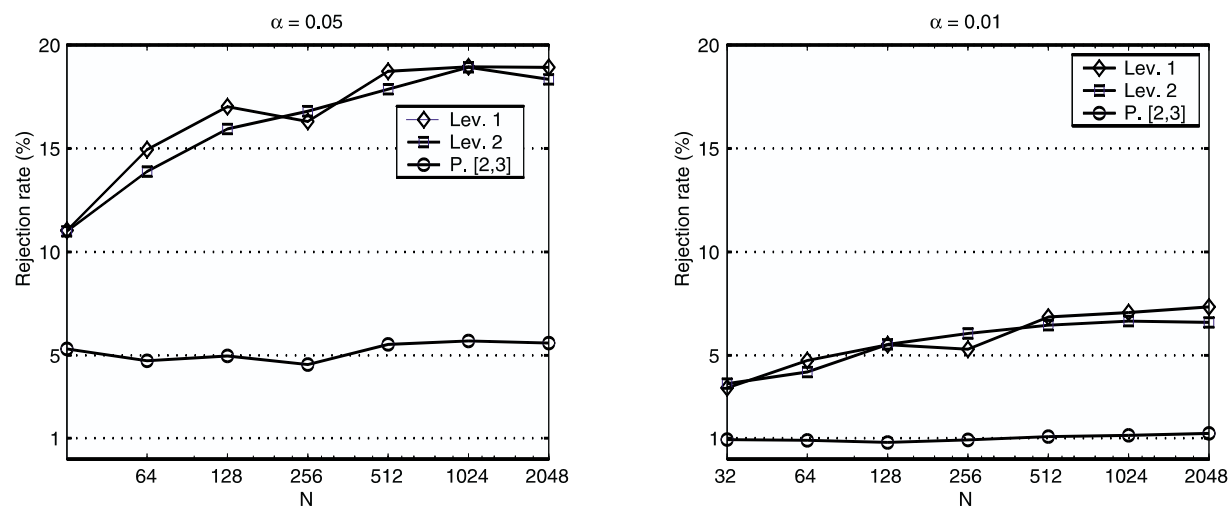

Figure 3. ARMA(2,2): Empirical size of the ICSS algorithm with level 1 DWT, level 2 DWT, and packet [2,3] DWPT coefficients.

rejection rates are very close to the values of $5 \%$ and $1 \%$.

Results from the study of the empirical power of the ICSS procedure are shown in Table 2. They were obtained with 10,000 replicates of the ICSS algorithm on time series from process (5.1) with fixed length $N=2,048$ and one variance change point at $k=1,024$. The parameter $\Delta$ indicates the ratio of the two variance values used to simulate the change point. When using DWT coefficients, due to the presence of correlation among the coefficients, the procedure tends to overestimate the number of variance change points. For example, if the ratio of the two variances is $\Delta=3$, two or more change points are identified in $21.6 \%$ of the replicates with level 1 DWT coefficients. The rejection rates for the case of one change point obtained with packet $[2,3]$ DWPT coefficients are, instead, always around $90 \%$, and moderately worse when $\Delta=1.5$.

We also exploited performances of the procedure in the detection of multiple change

Table 2. ARMA(2,2): Empirical Power of the ICSS Algorithm for $N=2,048$ and One Variance Change Point at $k=1,024$

\begin{tabular}{ccccr}
\hline \hline Level & \multicolumn{1}{c}{$\Delta$} & \multicolumn{1}{c}{0} & \multicolumn{1}{c}{1} & $\geq 2$ \\
\hline 1 & 1.5 & 4.2 & $\mathbf{7 8 . 7}$ & 7.1 \\
2 & 1.5 & 18.1 & $\mathbf{7 0 . 8}$ & 11.2 \\
2,3 & 1.5 & 19.4 & $\mathbf{7 5 . 5}$ & 5.1 \\
& & & & \\
1 & 2.0 & 0.7 & $\mathbf{7 9 . 5}$ & 19.8 \\
2 & 2.0 & 1.8 & $\mathbf{8 0 . 3}$ & 18.0 \\
2,3 & 2.0 & 0.9 & $\mathbf{9 3 . 2}$ & 5.9 \\
& & & & \\
1 & 3.0 & 0.5 & $\mathbf{7 7 . 9}$ & 21.6 \\
2 & 3.0 & 0.5 & $\mathbf{8 1 . 3}$ & 18.2 \\
2,3 & 3.0 & 0.6 & $\mathbf{9 2 . 4}$ & 7.1 \\
& & & & \\
1 & 10.0 & 0.7 & $\mathbf{7 7 . 7}$ & 21.7 \\
2 & 10.0 & 0.9 & $\mathbf{7 9 . 9}$ & 19.3 \\
2,3 & 10.0 & 0.8 & $\mathbf{8 9 . 7}$ & 9.5 \\
\hline & & & & \\
\end{tabular}


Table 3. Model (5.2): Empirical Power of the ICSS Algorithm for $N=5,840$ and Seven Variance Change Points

\begin{tabular}{ccccccr}
\hline \hline Level & $\Delta$ & $<6$ & 6 & 7 & 8 & $>8$ \\
\hline 1 & 10 & 2 & 1.5 & $\mathbf{2 8 . 8}$ & 26 & 41.7 \\
3,6 & 10 & 8.3 & 9.7 & $\mathbf{7 3}$ & 8.6 & 0.4 \\
\hline
\end{tabular}

points by using a more complicated process given by the summation of a sinusoidal component with frequency $1 / 1460$, a sinusoidal component with frequency $1 / 4$ and an $\operatorname{ARMA}(4,4)$ model

$$
\left(1-0.5 B+0.4 B^{2}-0.1 B^{3}-0.2 B^{4}\right) X_{t}=\left(1-0.2 B+0.1 B^{4}\right) \varepsilon_{t} .
$$

This model mimics some of the features of the measurements of crack widths that we will later analyze. We used $N=5,840$ and seven change points at $k_{1}=900, k_{2}=1,460, k_{3}=$ $2,360, k_{4}=2,920, k_{5}=3,820, k_{6}=4,380, k_{7}=5,280$. The ratio between variances was set to $\Delta=10$.

Rejection rates with Haar wavelets for the null hypothesis of no change points are shown in Table 3. They were obtained with 5,000 replicates of the ICSS algorithm. We also show the estimated locations of the variance change points in Figures 4 and 5. Level 1 DWT coefficients are heavily autocorrelated, and this causes change points to be overestimated. Eight or more change points are identified in $68 \%$ of the replicates. At packet $[3,6]$, chosen by the Ljung-Box test procedure, and corresponding to the frequency band [0.375, 0.4375], the algorithm correctly identifies seven change points in $73 \%$ of the replicates, and there is only a very small tendency to underestimate the number of change points. Also, the estimates obtained from the level 1 DWT are more spread out around the true change point locations, as can be seen from Figures 4 and 5 .

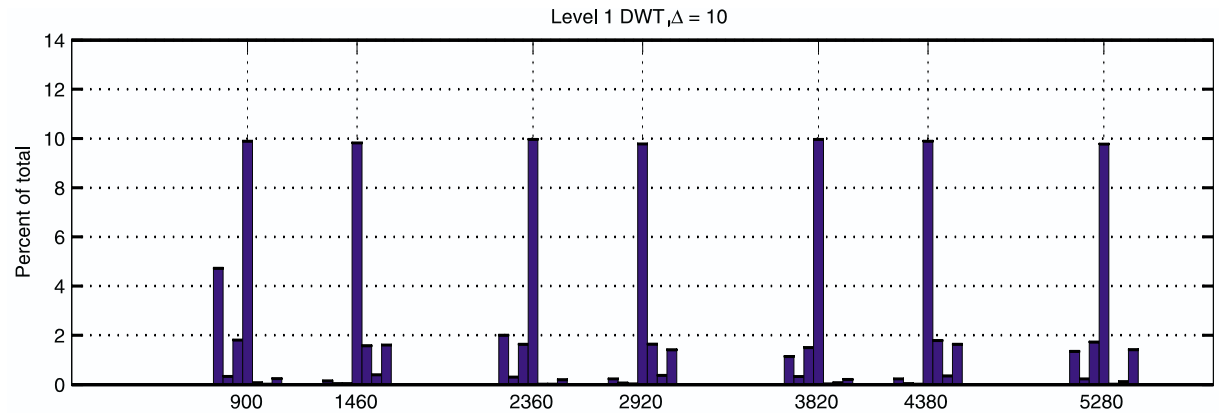

Figure 4. Model (5.2): Locations of variance change points estimated at level $1 \mathrm{DWT}$, for $N=5,840$ and seven change points. 


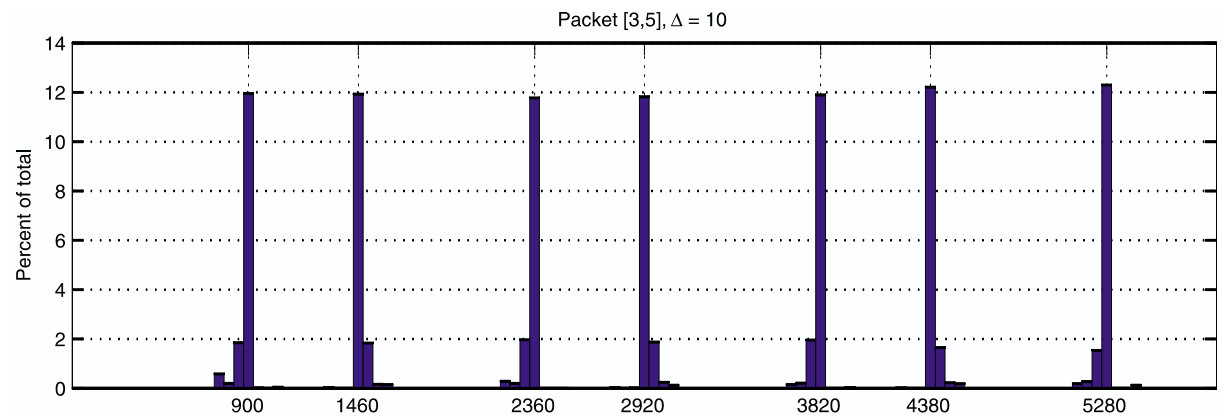

Figure 5. Model (5.2): Locations of variance change points estimated at packet [3,6] DWPT, for N=5,840 and seven change points.

\subsection{Subtidal Sea LeVels}

Percival and Mofjeld (1997) studied a time series of subtidal coastal sea levels at Crescent City, CA. The measurements are transmitted by a permanent tide gauge every six minutes. The data, shown in the top plot of Figure 7, are low-passed and subsampled every $1 / 2$ day. They range from the beginning of 1980 to the end of 1991 , for a total of $N=8,746$ observations. Percival and Mofjeld (1997) computed a time-dependent wavelet variance assuming that the wavelet coefficients are a portion of a realization of a process with constant variance in time segments of a given number of consecutive observations. They concluded that the variance of the series is large during the winter and small during the summer.

Our analysis revealed that packet [2,2] Haar coefficients, corresponding to the frequency interval [0.25, 0.375], are uncorrelated, as it appears from the Ljung-Box test statistic shown in Table 4 and from the autocorrelation functions shown in Figure 6. DWT coefficients at levels 1 and 2 were, instead, autocorrelated. The ICSS algorithm applied to packet [2,2] coefficients detected 36 variance change points, therefore partitioning the time series in 37 intervals where the variance can be considered constant. The vertical lines in Figure 7 indicate the estimated locations of the variance change points. The middle plot shows estimates of the packet [2,2] wavelet variance in the intervals defined by the variance change points, together with $95 \%$ confidence intervals; the bottom plot shows the level [2,2] MODWPT coefficients. The ICSS procedure nicely isolates intervals with similar variance. It clearly shows that the variance is periodic, with a fairly regular evolution, and that it is largest during the winter, generally from November until April.

Table 4. Subtidal Sea Levels: Ljung-Box Q Statistic for DWPT Coefficients at Level 2. Critical values are in parentheses.

\begin{tabular}{lccc}
\hline \hline Level & $Q_{10}$ & $Q_{20}$ & $Q_{30}$ \\
\hline 2,0 & $6311.20(18.31)$ & $9655.61(31.41)$ & $11309.60(43.77)$ \\
2,1 & 128.35 & 153.85 & 163.54 \\
2,2 & 13.76 & 25.80 & 39.19 \\
2,3 & 172.36 & 202.00 & 215.13 \\
\hline
\end{tabular}



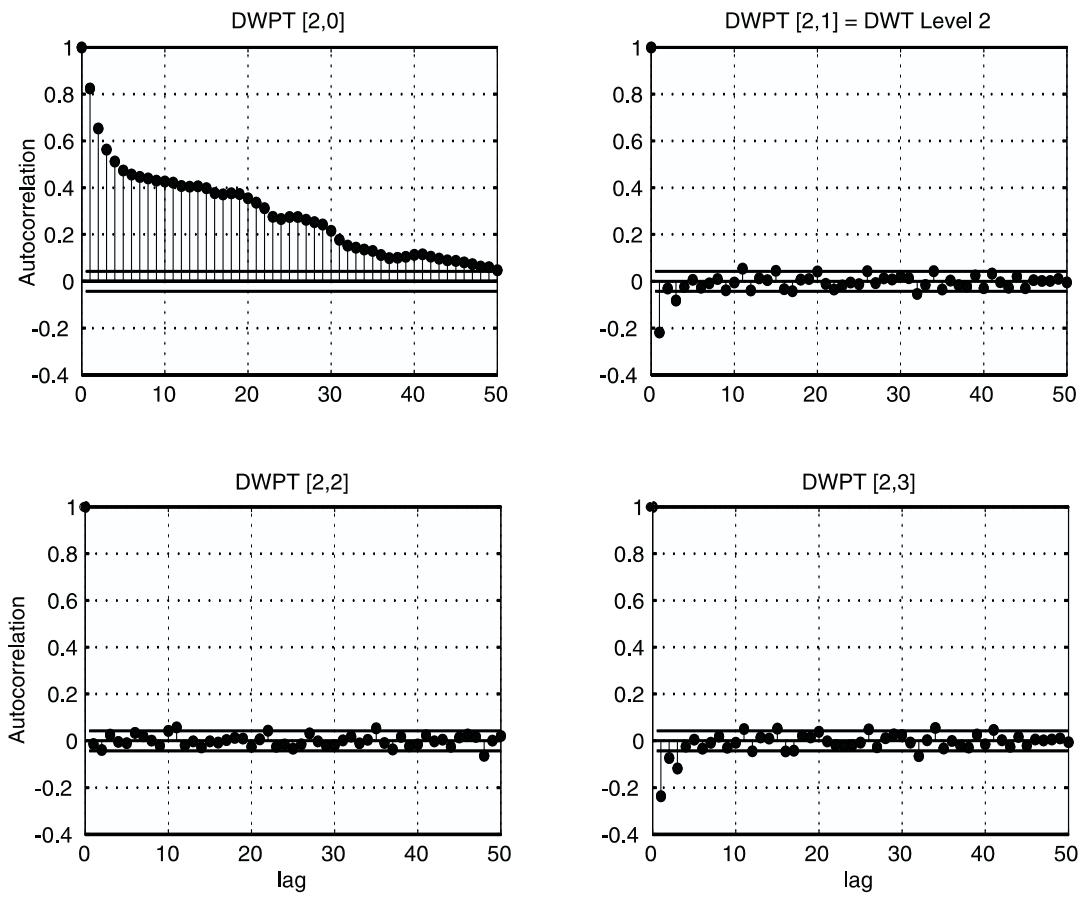

Figure 6. Subtidal sea levels: Autocorrelation functions at level 2 DWPT.
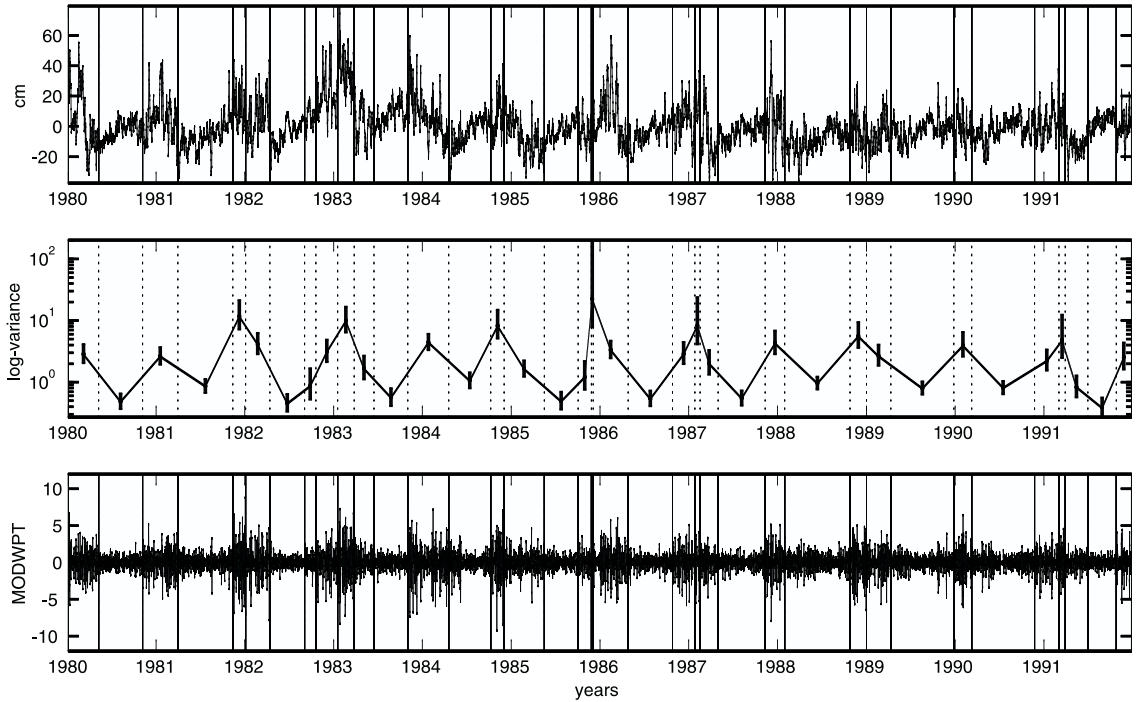

Figure 7. Subtidal sea levels. Top: data. Middle: estimates of packet [2,2] wavelet variance, on a log scale, in the intervals defined by the variance change points, with a 95\% C.I. Bottom: packet [2,2] MODWPT coefficients. Bars indicate the estimated locations of variance changes. Location indices are reported on the $x$-axes of top and middle plots. 


\subsection{Crack Widths in the Brunelleschi Dome}

The dome of Santa Maria del Fiore cathedral was designed by Filippo Brunelleschi, who also directed its construction (1434-1472). The structure includes an internal thick dome, with a structural support function, and an external thin one, which offers protection from atmospheric agents, such as rain. The domes are linked by several joining elements at the edges between adjacent webs and inside the webs themselves. The first cracks appeared soon after the construction was completed, the main cause being the dome itself, its weight and the insufficient resistance of the tambour; see Chiarugi, Fanelli, and Giuseppetti (1983). If we assign numbers to the eight webs of the dome, from 1 to 8 , with 1 being the web in front of the nave, at the present time the main cracks are in the even webs. They start from the tambour and stretch to the higher part of the dome, passing through both the internal and external dome.

Several control devices have been installed to monitor the evolution of the cracks. The most recent is a large digital monitoring system installed on January 8, 1988, that includes 166 instruments recording four measurements per day. Figure 8 is a schematic representation of the dome and its structural elements, together with the positions of the instruments located in web number four. Measured variables are temperatures (in the air and in the masonry) and crack widths. Crack widths are recorded by induction deformometers, whose edges are fixed up to the dome walls across the cracks, and are relative to the day in which the system began working, so that all time series exhibit a zero value at 00:00 of January 8, 1988. Deformometers record negative variations when the cracks are getting wider. Because of errors in the calibration of the instruments, measurements taken during the entire first year (1988) have been discarded.

We report here some details of the analysis of three instruments in the main crack: df406 (deformometer number six in web four, measuring crack variations in the inner part of the inner dome), df407 (located in the outer part of the inner dome) and df408 (in the inner part of the outer dome). Measurements are in centimeters and data are shown in the top plots of Figures 9, 10, and 11. Let us first consider instrument df406. With Haar wavelets the LjungBox test identified wavelet packet coefficients at packet $[3,6]$, corresponding to the time interval of approximately 13 to 16 hours, as a series of uncorrelated DWPT coefficients. The ICSS algorithm then located 13 variance change points, defining 14 intervals with constant variance. In each interval an estimate of the wavelet packet variance can be computed via Equation (3.6). This is shown in Figure 9. The intraday variance appears seasonally dependent. It increases during spring, achieves its maxima in the summer, and the minima in winter. The increased variability of crack width variations in the summer can be explained by the fact that, as the temperature increases, the building materials dilate and, as the dome is tied up at its bottom and at its top, the crack width is forced to decrease its width. In winter the opposite happens, and the process has greater variance when the crack is closing because in this case the two edges of the crack press each other. From the top plot of Figure 9 we can also notice that the crack width gets close to its minimum during the summer. 

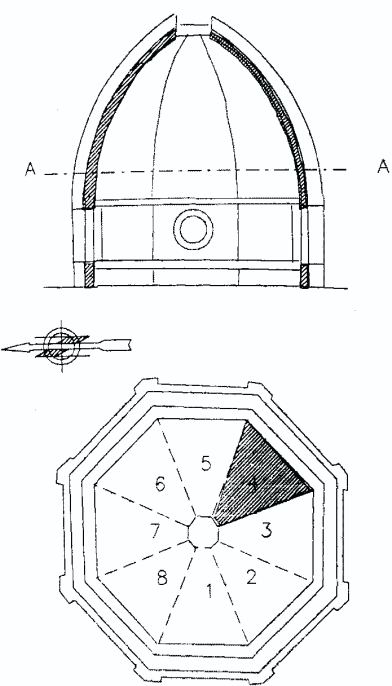

SEZ , A-M A

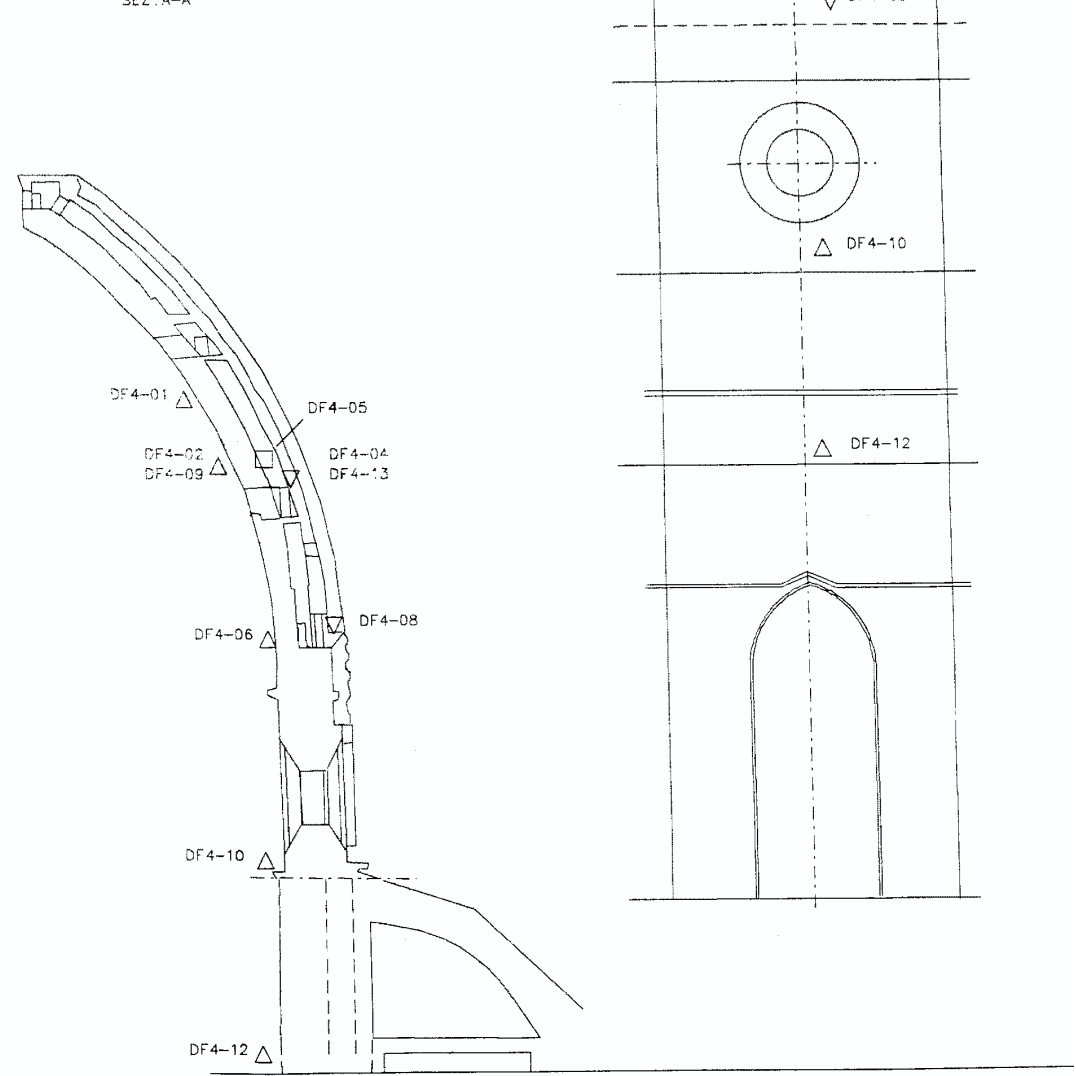

KEY TO SYMBOLS

$\triangle$ DEFORMOMETERS - INNER SIDE OF INTERNAL DOME

$\Delta$ DEFORMOMETERS - OUTER SIDE OF INTERNAL DOME
$\nabla$ DEFORMOMETERS - INNER SIDE OF EXTERNAL DOME

Figure 8. Brunelleschi dome: Schematic representation of the structural elements of the dome and of the locations of the instruments located in the web number 4. 

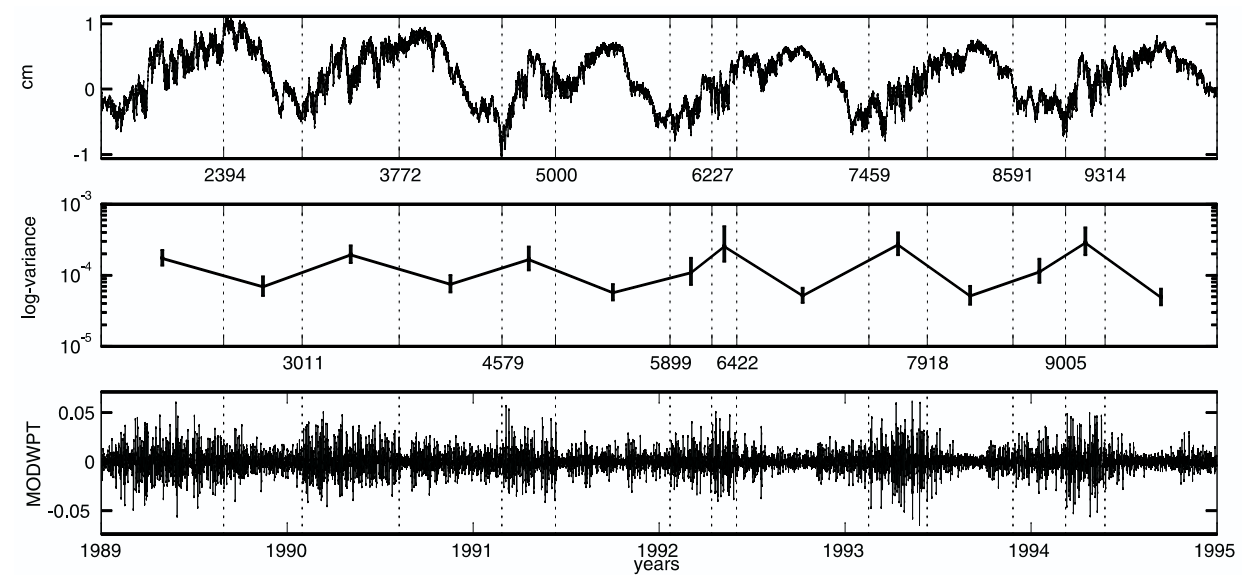

Figure 9. Crack widths in the Brunelleschi dome, deformometer df406 (number 6 in web 4). Top: data. Middle: estimates of packet [3,6] wavelet variance, on a log scale, in the intervals defined by the variance change points, with a 95\% C.I. Bottom: packet [3,6] MODWPT coefficients. Bars indicate the estimated locations of variance changes. Location indices are reported on the $x$-axes of top and middle plots.
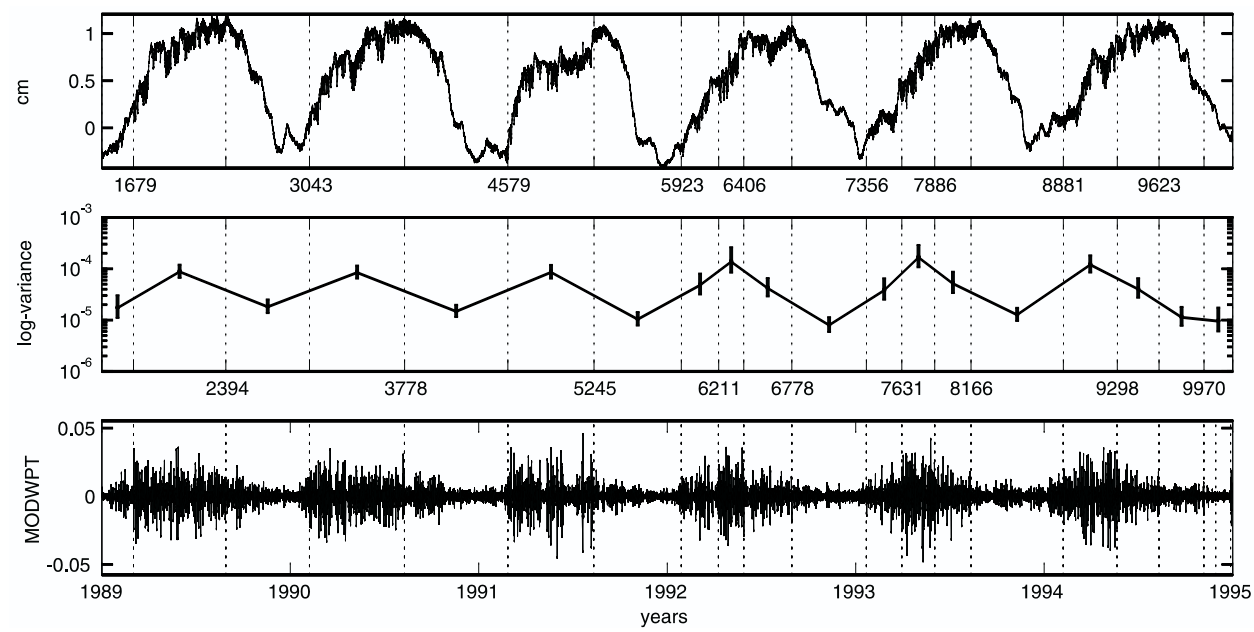

Figure 10. Crack widths in the Brunelleschi dome, deformometer df407. Top: data. Middle: estimates of packet [3,5] wavelet variance, on a log scale, in the intervals defined by the variance change points, with a 95\% C.I. Bottom: packet [3,5] MODWPT coefficients. Bars indicate the estimated locations of variance changes. Location indeces are reported on the $x$-axes of top and middle plots. 

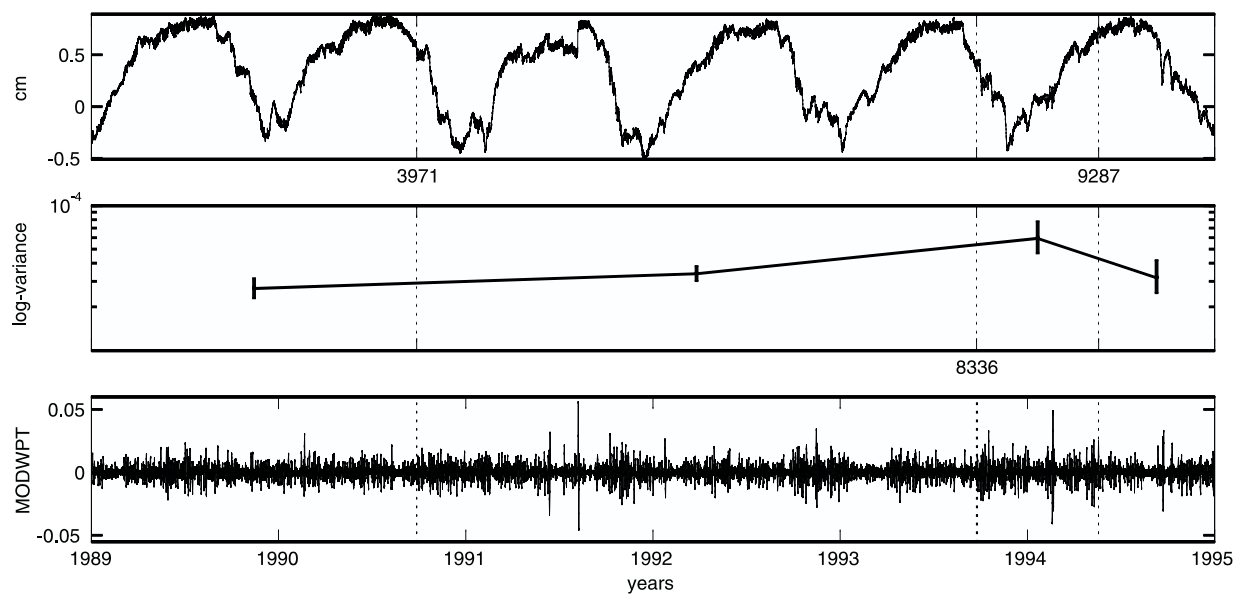

Figure 11. Crack widths in the Brunelleschi dome, deformometer df408. Top: data. Middle: estimates of packet [3,2] wavelet variance, on a log scale, in the intervals defined by the variance change points, with a 95\% C.I. Bottom: packet [3,2] MODWPT coefficients. Bars indicate the estimated locations of variance changes. Location indices are reported on the $x$-axes of top and middle plots.

As for instrument df407, change points were identified using wavelet packet coefficients at level [3,5], corresponding to the frequency band [0.3125, 0.375] and to a time interval of approximately 16 to 19 hours, and follow a pattern similar to deformometer df406 (see Figure 10). The variance achieves its maxima in the summer and its minima in the winter. It is important to notice that the wavelet packet variance for instrument $\mathrm{df} 407$ is overall lower than the variance for df406, and also that the difference between minima and maxima is more relevant. For instrument df408, instead, measurements show a considerably different behavior (see Figure 11), in that they exhibit no significant seasonality.

From the analysis of the three instruments we notice a tendency of the variance to get lower when moving towards the outer part of the dome. This was also confirmed by the analysis of instruments on other webs and can be explained by the different role that the outer and the inner dome play, the latter having a structural support function and thus having to support the weight of the entire monument. Another interesting feature that was highlighted by the comparison of instruments at different latitudes on the main crack of web four is that measurements coming from the instruments located near the tambour of the dome have a smaller intraday variance than the ones located on the top. Instrument df412 (being the closest instrument to the tambour) showed no annual seasonality. An explanation for this could lie in the fact that the dome is tied up in two points: at its top by the lantern, and, more steadily, at its bottom, where the main nave of the church and the three side chapels greatly limit its movements. Similar analyses performed with data collected by instruments located on the main cracks in web six confirmed these observations.

\section{DISCUSSION}

In this article we have used wavelet packets to extend recent wavelet approaches to variance estimation for time series. First, we have defined the wavelet packet variance as a 
generalization of the wavelet variance of Percival (1995). Results reported in the supplementary material show that our estimator of the packet variance is unbiased and asymptotically Gaussian when the underlying process is Gaussian. These results are generalizations of those presented by Percival (1995) and Percival and Walden (2000) for the wavelet variance. Proofs are based mainly on the fact that wavelet packet filters $\left\{\tilde{f}_{j, n, l}\right\}$ can be computed from wavelet and scaling filters through Equations (3.3) and (3.2) and that the squared gain function of wavelet packet filters can be obtained as a product of those of scaling and wavelet filters. Serroukh et al. (2000) have generalized some results about the distribution of the wavelet variance estimator to processes that are not necessarily Gaussian and, based on the generality of their results and proofs, we expect similar extensions to be possible for the wavelet packet variance.

For the case of processes with nonconstant variance we have adapted to wavelet packets the ICSS procedure of Inclàn and Tiao (1994) to identify variance change points. There the test statistic is computed on wavelet packet coefficients, while the location of the variance change points is estimated by nondecimated wavelet packet coefficients. We have shown via simulations how this procedure can be applied to time series coming from a wide class of stochastic processes and have studied empirical size and power of the testing procedures. In selecting the wavelet packet to be subjected to the ICSS algorithm strategies other than the one we have adopted can be explored, perhaps by identifying and combining multiple sets of packets at increasing levels for which one fails to reject the white noise test. Notice, however, that simulations have suggested that the size and power of the ICSS procedure decrease with the wavelet level and that this may affect the results. For future work possible $p$ values adjustments for the Box-Ljung test could also be considered, to compensate for multiple comparisons.

We have applied wavelet packet techniques to time series collected in different fields, first to a time series of subtidal coastal sea levels, previously studied by Percival and Mofjeld (1997) using the wavelet variance, and then to time series of crack width variations recorded in the Brunelleschi dome in Florence. Examples have highlighted the great potential of wavelet packet variance techniques as exploratory tools for time series analysis. In the latter example, our analysis has revealed some interesting aspects regarding the dynamics of crack evolutions and the structural functions of the different elements of the dome. It is expected that crack width variations will be influenced in some way by the temperature. The relation between these two phenomena is quite complex and will require further analysis. In Gabbanini (2002), based on the exploratory analyses with the wavelet packet variance here presented, time series modeling is investigated. Best results are found with ARMAARCH models, Bollerslev (1986) and Engle (1982), fitted to the differences of variations of cracks measured at different depths (but at the same height and on the same crack) in the masonry with the thermal gradient as an explanatory variable. Such models adequately describe dynamics that are well known to engineers. See Gabbanini (2002) for more details.

\section{ACKNOWLEDGMENTS}

The authors thank the AE and three anonymous referees for their careful reading and for the many suggestions that led to a substantially improved version of this paper. Marina Vannucci is partially supported by National Science 
Foundation, CAREER award number DMS-0093208, by the Texas Higher Education Advanced Technology Program, and by Telecommunications and Informatics Task Force at TAMU.

\section{[Received August 2002. Revised June 2003.]}

\section{REFERENCES}

Bollerslev, T. (1986), “Generalized Autoregressive Conditional Heteroskedasticity,” Journal of Econometrics, $\underline{31,307-327 .}$

Box, G. E. P., Jenkins, G. M., and Reinsel, G. C. (1994), Time Series Analysis: Forecasting and Control (3rd ed.), Englewood Cliffs, NJ: Prentice Hall.

Chiarugi, A., Fanelli, M., and Giuseppetti, G. (1983), "Analysis of a Brunelleschi-Type Dome Including Thermal Loads," in IABSE Symposium on Strenghtening of Building Structure, Diagnosis and Therapy, Zurich, pp. $169-178$.

Coifman, R. R., and Donoho, D. (1995), “Time Invariant Wavelet Denoising,” in Wavelets and Statistics, eds. A. Antoniadis and G. Oppenheim, Lecture Notes in Statistics, vol. 103, New York: Springer, pp. 125-150.

Daubechies, I. (1992), Ten Lectures on Wavelets (vol. 61), Philadelphia: SIAM.

Engle, R. F. (1982), “Autoregressive Conditional Heteroskedasticity With Estimates of the Variance of United Kingdom Inflation,” Econometrica, 50, 987-1007.

Gabbanini, F. (2002), "Analisi dei dati Provenienti dal Sistema di Monitoraggio Installato Sulla Cupola di Santa Maria del Fiore," unpublished Ph.D. thesis, Department of Statistics, University of Florence, Italy.

Inclàn, C., and Tiao, G. C. (1994), "Use of Cumulative Sums of Squares for Retrospective Detection of Changes in Variance," Journal of the American Statistical Association, 89, 913-923.

Mallat, S. G. (1989), “A Theory of Multiresolution Signal Decomposition: The Wavelet Representation,” IEEE Transactions on Pattern Analysis and Machine Intelligence, 11, 674-693.

Nason, G. P., and Silverman, B. W. (1995), “The Stationary Wavelet Transform and Some Statistical Applications," in Wavelets and Statistics, eds. A. Antoniadis and G. Oppenheim, Lecture Notes in Statistics, vol. 103, New York: Springer, pp. 281-300.

Percival, D. B. (1995), “On the Estimation of the Wavelet Variance,” Biometrika, 82, 619-631.

Percival, D. B., and Mofjeld, H. (1997), “Analysis of Subtidal Coastal Sea Level Fluctuations Using Wavelets," Journal of the American Statistical Association, 92, 868-880.

Percival, D. B., and Walden, A. T. (2000), Wavelet Methods for Time Series Analysis, Cambridge: Cambridge University Press.

Percival, D. B., Sardy, S., and Davison, A. C. (2000), "Wavestrapping Time Series: Adaptive Wavelet-Based Bootstrapping," in Nonlinear and Nonstationary Signal Processing, eds. B. J. Fitzgerald, R. L. Smith, A. T. Walden, and P. C. Young, Cambridge, UK: Cambridge University Press.

Serroukh, A., Walden, A. T., and Percival, D. B. (2000), "Statistical Properties and Uses of the Wavelet Variance Estimator for the Scale Analysis of Time Series," Journal of the American Statistical Association, 95 , $184-196$.

Shensa, G. (1992), "The Discrete Wavelet Transform: Wedding the à trous and Mallat Algorithms," $\underline{I E E E}$ Transactions on Signal Processing, 40, 2464-2482.

Tewfik, A. H., and Kim, M. (1992), "Correlation Structure of the Discrete Wavelet Coefficients of Fractional Brownian Motion," IEEE Transactions on Information Theory, 38, 904-909.

Walden, A. T., and Contreras Cristan, A. (1998), “The Phase-Corrected Undecimated Discrete Wavelet Packet Transform and its Application to Interpreting the Timing of Events," Proceedings of the Royal Society of London, A, 454, pp. 2243-2266. 
Whitcher, B., Byers, S. D., Guttorp, P., and Percival, D. B. (2002), “Testing for Homogeneity of Variance in Time Series: Long Memory, Wavelets and the Nile River," Water Resources Research, 38, 10.1029/2001WR000509.

Whitcher, B., Guttorp, P., and Percival, D. B. (2000), "Multiscale Detection and Location of Multiple Variance Changes in the Presence of Long Memory," Journal of Statistical Computation and Simulation, 68, 65-88.

Wickerhauser, M. V. (1994), Adapted Wavelet Analysis from Theory to Software Algorithms, Wellesley, MA: AK Peters. 